\title{
Generalization Ability of Dynamic Systems by Using Second Order Derivatives of Universal Learning Network
}

$\begin{array}{lll}\text { Student Member } & \text { Min Han } & \text { (Kyushu University) } \\ \text { Member } & \text { Kotaro Hirasawa } & \text { (Kyushu University) } \\ \text { Member } & \text { Jinglu Hu } & \text { (Kyushu University) } \\ \text { Member } & \text { Junichi Murata } & \text { (Kyushu University) }\end{array}$

This paper studies how the generalization ability of modeling of the dynamic systems can be improved by taking advantages of second order derivatives of the criterion function with respect to the external inputs that can be considered as a direct implementation of the well-known regularization technique. Computation of second order derivatives of Universal Learning Network for a dynamic network are discussed. Simulation studies of a nonlinear dynamic system and a real robot system are carried out to demonstrate the effectiveness of the proposed method. Simulation results show that the proposed method can improve the generalization ability of neural networks sufficiently by selecting an appropriate regularization parameter.

Key words: Generalization Ability, Universal Learning Network, Regularization Technique, Second Order Derivatives

\section{Introduction}

Since the first proposal of a neuron model by $\mathrm{McCul}-$ loch and Pitts in 1940's, especially after the revitalization of artificial neural networks in 1980's, a variety of neural networks have been devised and are now applied in many fields. The vast majority of neural networks in use are those networks whose parameters or weights are tuned by the gradient-based supervised learning. This category includes feedforward neural networks or multilayer perceptrons, various types of recurrent neural networks. These networks seemingly have different architectures and are trained by distinguishable training algorithms. In essence, however, they can be unified in a single framework in regard to both their architectures and learning algorithms.

Universal Learning Networks (ULNs) ${ }^{(1)(2)}$ have been proposed, as the name indicates, to provide a universal framework for the class of neural networks. Unification of a variety of neural architectures and their learning algorithms is an objective of ULNs, this provides a consistent viewpoint for the various kinds of neural networks. However, there is another benefit that is expected for ULNs.

Generalization of the architectures and the gradientbased learning algorithms does not only give a unified description but attains new abilities of the networks. Allowing high degrees of freedom in their architectures gives them more flexibilities and representing abilities, and therefore the ULNs can be useful and effective tools for modeling and controlling large-scale nonlinear complex systems including physical, social and economical phenomena. In addition to calculation of the first or- der derivatives of the signals flowing in the networks that are necessary in the gradient-based learning, the generalized ULNs learning algorithm is equipped with a systematic mechanism that calculates their second or higher order derivatives ${ }^{(3)}$. This allows elaborate criterion functions to be used in their learning, which enables more sophisticated specification of the network performance.

On the other hand, generalization ability of neural networks is known to be a very important characteristics of neural networks, which improves the adaptability of neural networks to practical problems.

Generally speaking, there are two kinds of methods to improve the generalization ability of the networks. One method is to increase the volume of training data. Other method called regularization method is to decrease the degree of freedom of the networks.

As for the regularization method, where a second term is added to the original criterion function, there have been reported many papers such as weight decay method $^{(4)}$, structural learning method ${ }^{(5)}$ and weight elimination method ${ }^{(6)}$.

All these methods use the term composed of parameters as the second term in the criterion function. And also Poggio has presented a method ${ }^{(7)}$ which can deal with the more general second term represented by differential operation.

This paper is an extension of the Poggio's method in terms that the networks is extended to dynamical systems from static systems, and the second order derivatives of the criterion function with respect to parameters are used in order to adjust the parameters.

In section 2, the Universal Learning Network is outlined. Section 3 proposes the method for improving the 


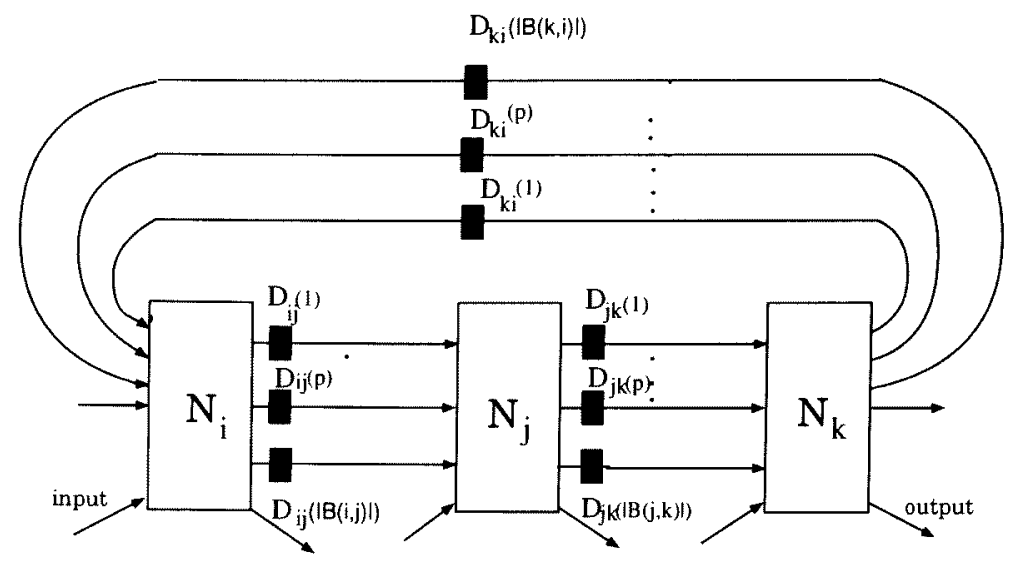

Fig.1 Structures of ULN with multi-branches

generalization ability of the dynamic systems by introducing the second term defined by the deviation of the criterion function caused by the external input changes. Training algorithm of parameters are described in section 4 using the higher order derivatives of ULNs. Identification problems of a nonlinear dynamic system and a real robot system are dealt with in section 5 as examples, where it is shown that more robust identifications of the nonlinear dynamic system are realized by the proposed method. Section 6 gives conclusions.

\section{ULN Structure ${ }^{(3)}$}

As stated in the introduction, a distinctive feature of the proposed method is to use the higher order derivatives of ULNs to improve the generalization ability. In this section, ULNs is briefly described while calculation method of the higher order derivatives will appear in section 4

The structure of ULNs with multi-branches ${ }^{(8)(9)}$ is shown in Fig.1.

The ULNs consists of a number of nodes and multibranches for inter-connecting the nodes. The nodes may have any continuously differentiable nonlinear functions in them and each pair of nodes can be connected each other by multi-branches with arbitrary (positive, zero or even negative) time delays. ULNs including negative time delay can be used to model systems that have prediction mechanisms in them.

The general equation that describes the behavior of ULNs is represented by Eq.(1),

$$
\begin{array}{r}
h_{j}(t)=f_{j}\left(\left\{h_{i}\left(t-D_{i j}(p)\right) \mid i \in J F(j), p \in B(i, j)\right\},\right. \\
\left.\left\{r_{n}(t) \mid n \in N(j)\right\},\left\{\lambda_{m}(t) \mid m \in M(j)\right\}\right) \\
j \in J, t \in T \cdots \cdots \cdots(1)
\end{array}
$$

where

$h_{j}(t)$ : output value of node $j$ at time $t$,

$\lambda_{m}(t)$ : value of $m$-th parameter at time $t$,

$r_{n}(t)$ : value of $n$-th external input variable at time $t$,

$f_{j}(\cdot)$ : nonlinear function of node $j$,

$D_{i j}(p)$ : time delay of $p$-th branch from node $i$ to node $j$

$J F(j)$ : set of nodes which are connected to node $j$,

$B(i, j)$ : set of branches from node $i$ to node $j$

$N(j):\left\{n \mid r_{n}\right.$ is fed to node $\left.j\right\}$,

$N$ : set of external input variables $\{n\}$,

$M(j):\left\{m \mid h_{j}\right.$ is partially differentiable with respect to $\left.\lambda_{m}\right\}$,

$M$ : set of parameters $\{m\}$,

$J$ : set of nodes $\{j\}$,

$T$ : discrete set of sampling instants.

In the Universal Learning Network, Eq.(1) can be applied to not only dynamic systems but also static systems eliminating the sampling $t$ and time delay $D_{i j}(p)$.

The ULNs operates on a discrete-time basis. Functions $f_{j}($.$) that govern the operation of the nodes can$ be any continuously differentiable functions; typically, sigmoid functions can be employed. And in this case, Eq.(1) can be expressed specifically as follows,

$$
\begin{aligned}
& h_{j}(t)=f_{j}\left(\alpha_{j}(t)\right)=\frac{1}{1+e^{-\varphi_{j} \alpha_{j}(t)}} \cdots \cdots \cdots \\
& \alpha_{j}(t)=\sum_{i \in J F(j)} \sum_{p \in B(i, j)} w_{i j}(p) h_{i}\left(t-D_{i j}(p)\right)+\theta_{j}
\end{aligned}
$$

where,

$$
\begin{gathered}
w_{i j}(p): \text { weight paramter of } p \text {-th branch } \\
\text { from node } i \text { to node } j \\
\theta_{j}: \text { threshold parameter of node } j \\
\varphi_{j}: \text { slope parameter of node } j .
\end{gathered}
$$

\section{Regularization Technique}

Regularization technique is well known in approxima- 
tion theory and has been proved to be useful, see e.g. Ref. (10). In this section, we will introduce a direct implementation of the regularization technique to ULN training.

\subsection{Regularization Networks}

Regularization theory can be described as follows: Let $S=\left\{\left(x_{i}, y_{i}\right) \in R^{d} \times R \mid i=1, \ldots, N\right\}$ be a set of data that we want to approximate by means of a function $f$. The regularization approach ${ }^{(11)-(14)}$ consists in looking for the function $f$ that minimizes the functional

$$
L[f]=\sum_{i=1}^{N}\left(y_{i}-f\left(x_{i}\right)\right)^{2}+\lambda\|P f\|^{2}
$$

where $f: R^{d} \rightarrow R, x_{i} \in R^{d}$ is the input, $y_{i} \in R$ is the output, $N$ is the number of training data sets, $P$ is a constraint operator (usually a differential operator), $\|\cdot\|$ is a norm on the function space to whom $f$ belongs (usually the $\mathcal{L}^{2}$ norm) and $\lambda$ is a positive real number, the so called regularization parameter. The structure of the operator $P$ embodies the a priori knowledge about the solution, and therefore depends on the nature of the particular problem that has to be solved.

Minimization of the functional $L$ leads to the associated Euler-Lagrange equations. By solving the EulerLagrange equations, a class of three-layer networks called regularization networks were obtained ${ }^{(7)(10)}$. The most general form of the regularization networks is called Hyper Basis Functions network that is closely related with the well-known Radial Basis Functions network. A regularization network is proved to be able to approximate arbitrarily well any multivariate continuous function on a compact domain, given a sufficiently high number of units. Moreover, because the approximation scheme derived from regularization theory is linear in the unknown coefficients, it can be proved that it has the so called best-approximation property ${ }^{(15)}$. However, a regularization network may become very complicated when data set $N$ is large.

\subsection{Network Training Techniques}

For a certain network with pre-determined $f$, for instance, sigmoid neural network, minimizing $L$ leads to a computation of higher order derivatives when $P$ is a differential operator. Computing higher order derivatives is usually considered to be complicated and timeconsuming. Some schemes have been proposed to avoid computing higher order derivatives. A typical example is to use $\lambda\left\|W-W_{0}\right\|^{2}$ as regularization term so that

$$
L[f]=\sum_{i=1}^{N}\left(y_{i}-f\left(\boldsymbol{x}_{i}\right)\right)^{2}+\lambda\left\|W-W_{0}\right\|^{2} \cdots(5)
$$

where $W$ is the vector of unknown parameters and $W_{0}$ is the true value which is unknown and practically replaced using some normal guess $W^{\#(16)}$. When the normal guess $W^{\#}$ is simply chosen to be 0 , the regularization term becomes

$$
\lambda\left\|W-W_{0}\right\|^{2}=\lambda\|W\|^{2}=\lambda \sum_{i, j} \omega_{i, j}^{2}
$$

which can obviously be considered as an effort to reduce the effect of redundant parameters of networks and to make it compact. Other well-known methods for reducing the effect of redundant parameters and making a network compact include:

(1) forgetting learning method ${ }^{\{17\}}$

$$
L[f]=\sum_{i=1}^{N}\left(y_{i}-f\left(\boldsymbol{x}_{i}\right)\right)^{2}+\lambda \sum_{i, j}\left|\omega_{i, j}\right| \cdots \cdots
$$

that was claimed to be a structural learning method; (2) weight elimination method ${ }^{(6)}$

$$
L[f]=\sum_{i=1}^{N}\left(y_{i}-f\left(\boldsymbol{x}_{i}\right)\right)^{2}+\lambda \sum_{i, j} \frac{\left(\frac{\omega_{i j}}{\omega_{0}}\right)^{2}}{1+\left(\frac{\omega_{i j}}{\omega_{0}}\right)^{2}} \cdots
$$

where $\omega_{0}$ is the initial parameter value.

\subsection{Direct Implementation of Regularization Technique}

All the above three methods defined by Eq.(5),(6) and (7) try to reduce the effect of redundant parameters and make a network compact. They have been shown to be useful for improving generalization ability of networks.

However, a question arises here of whether "a network can be trained to benefit from the redundant parameters?." If we properly choose the differential operator $P$, the regularization term $\lambda\|P f\|^{2}$ may be interpreted as an effort to control the sensitivity of a network. In a neural network based system, a low sensitivity to a certain parameter means a high robustness to that parameter which is practically useful. Therefore, it is worth implementing the regularization term directly although it is rather time-consuming. This is the motivation of this paper.

Based on the regularization technique, let us consider the following criterion function for the ULN

$$
L=E+k_{d} E_{d}
$$

where $E=E\left(\left\{h_{r}(s)\right\}\right), r \in J_{0}, s \in T_{0}$ and

$$
E_{d}=\sum_{n \in N} \sum_{t^{\prime} \in T^{\prime}}\left(\frac{\partial^{\dagger} E}{\partial r_{n}\left(t^{\prime}\right)} \Delta r_{n}\left(t^{\prime}\right)\right)^{2}
$$

$J_{0}:\left\{r \mid h_{r}\right.$ is related to evaluation $\}$

$T_{0}:\{s \mid$ time instant related evaluation $\}$

$T^{\prime}:\left\{t^{\prime} \mid\right.$ time when $r_{n}\left(t^{\prime}\right)$ is changed $\}$

$k_{d}$ : regularization parameter

The first term $\mathrm{E}$ of Eq.(8) is the same basic term of the criterion function as Eq.(4), and the second term $E_{d}$ represents the deviation of the criterion function $E$ caused by the change of the external input variable $r_{n}\left(t^{\prime}\right)$ at time $t^{\prime}$. Minimizing Eq. (8) leads to a computation of second order derivatives. In the next section, 
a computing method of the second order derivatives of the criterion function $E$ with respect to parameter $\lambda_{m}$ and $r_{n}\left(t^{\prime}\right)$ will be discussed.

\section{Parameter Training}

Now, we shall introduce a new calculation scheme for the $n$-th order derivatives of the criterion function $\mathrm{E}$ by using the forward propagation method. In the forward propagation calculation, the changes of node outputs with respect to the changes in the parameters propagate forward in the networks, while the changes of the criterion function with respect to the changes in node outputs propagate backward in the backward propagation calculation usually used in neural network learning. The reason why the forward propagation method is adopted in this paper is due to lower computational load as described in reference (2). Details of the $n$-th order derivatives calculation by forward propagation method can be found in reference (2). In this section, only the calculation of the first and second order derivatives is discussed as examples.

First, let parameter $\lambda_{m}$ of ULN be defined as follows,

$$
\lambda_{m} \in\left\{w_{i j}(p) \mid j \in J, i \in J F(j), p \in B(i, j)\right\} .
$$

where $w_{i j}(p)$ is the adjustable parameters related to node $j$.

Training of ULN is realized by minimizing the criterion function $\mathrm{L}$ based on the gradient method

$$
\lambda_{m} \leftarrow \lambda_{m}-\gamma \frac{\partial^{\dagger} L}{\partial \lambda_{m}}
$$

where $\gamma$ is the learning coefficient assigned a small positive value and $\frac{\partial^{\dagger} L}{\partial \lambda_{m}}$ is the ordered derivatives defined by Werbos ${ }^{(18)}$. The head note $t$ means the change of the criterion function $\mathrm{L}$ caused by the change of $\lambda_{m}$ with other variables being fixed, where

$$
\frac{\partial^{\dagger} L}{\partial \lambda_{m}}=\frac{\partial^{\dagger} E}{\partial \lambda_{m}}+k_{d} \frac{\partial^{\dagger} E_{d}}{\partial \lambda_{m}}
$$

Ordered derivative $\frac{\partial^{\dagger} E}{\partial \lambda_{m}}$ can be obtained by the ordinary first order derivative calculation, but $\frac{\partial^{\dagger} E_{d}}{\partial \lambda_{m}}$ needs the second order derivative calculation because the following equation holds:

$$
\begin{aligned}
\frac{\partial^{\dagger} E_{d}}{\partial \lambda_{m}}= & 2 \sum_{n \in N} \sum_{t^{\prime} \in T^{\prime}}\left[\left(\frac{\partial^{\dagger} E}{\partial r_{n}\left(t^{\prime}\right)} \Delta r_{n}\left(t^{\prime}\right)\right)\right. \\
& \left.\times \frac{\partial^{\dagger 2} E}{\partial r_{n}\left(t^{\prime}\right) \partial \lambda_{m}} \Delta r_{n}\left(t^{\prime}\right)\right] \ldots \ldots .
\end{aligned}
$$

In the following subsections, the computation method of $\frac{\partial^{\dagger} E}{\partial r_{n}\left(t^{\prime}\right)}$, and $\frac{\partial^{\dagger 2} E}{\partial r_{n}\left(t^{\prime}\right) \partial \lambda_{m}}$ will be explained in detail.

\subsection{Computation of $\frac{\partial^{\dagger} E}{\partial r_{n}\left(t^{\prime}\right)}$}

In contrast to the backward propagation procedure described in the reference [1], in the forward propagation calculation, the derivatives of $E$ with respect to node output $h_{r}(s)$ are fixed, while the changes of node output $h_{r}(s)$ propagate through nodes.

In this paper, parameters are time invariant.

First order derivative of $E$ with respect to external input $r_{n}\left(t^{\prime}\right)$ can be written in the form of Eq.(13) assuming $t^{\prime}$ to be designated sampling instant,

$$
\begin{aligned}
\frac{\partial^{\dagger} E}{\partial r_{n}\left(t^{\prime}\right)} & =\sum_{r \in J_{o}} \sum_{s \in T_{o}}\left(\frac{\partial E}{\partial h_{r}(s)} \frac{\partial^{\dagger} h_{r}(s)}{\partial r_{n}\left(t^{\prime}\right)}\right) \\
& +\frac{\partial E}{\partial r_{n}\left(t^{\prime}\right)} \cdots \cdots \cdots \cdots \cdots \cdots \cdots
\end{aligned}
$$

As $\frac{\partial E}{\partial h_{r}(s)}$ and $\frac{\partial E}{\partial r_{n}\left(t^{\prime}\right)}$ can be calculated easily, it is important to calculate $\frac{\partial^{\dagger} h_{r}(s)}{\partial r_{n}\left(t^{\prime}\right)}$.

$\frac{\partial^{\dagger} h_{r}(s)}{\partial r_{n}\left(t^{\prime}\right)}$ can be generally expressed by the following iterative equation.

$$
\begin{aligned}
\frac{\partial^{\dagger} h_{k}(t)}{\partial r_{n}\left(t^{\prime}\right)}= & \sum_{j \in J F(k)} \sum_{p \in B(j, k)}\left(\frac{\partial h_{k}(t)}{\partial h_{j}\left(t-D_{j k}(p)\right)}\right. \\
& \left.\times \frac{\partial^{\dagger} h_{j}\left(t-D_{j k}(p)\right)}{\partial r_{n}\left(t^{\prime}\right)}\right)+\frac{\partial h_{k}(t)}{\partial r_{n}\left(t^{\prime}\right)} \cdots
\end{aligned}
$$

Therefore the ordered derivative of node output $h_{k}(t)$ with respect to the external input $r_{n}\left(t^{\prime}\right)$, that is, $P_{1}\left(k, t, r_{n}\left(t^{\prime}\right)\right)=\frac{\partial \dagger h_{k}(t)}{\partial r_{n}\left(t^{\prime}\right)}$, can be calculated by using the forward propagation procedure:

$$
\begin{aligned}
P_{1}\left(k, t, r_{n}\left(t^{\prime}\right)\right)= & \sum_{j \in J F(k)} \sum_{p \in B(j, k)}\left[\frac{\partial h_{k}(t)}{\partial h_{j}\left(t-D_{j k}(p)\right)}\right. \\
& \left.\times P_{1}\left(j, t-D_{j k}(p), r_{n}\left(t^{\prime}\right)\right)\right] \\
& +\frac{\partial h_{k}(t)}{\partial r_{n}\left(t^{\prime}\right)} \ldots \ldots \ldots \ldots \ldots \ldots \ldots \ldots \ldots \ldots
\end{aligned}
$$

In equation (15), the first term in the right-hand side indicates the indirect effect which is calculated taking the outputs of the upstream nodes $j$ as the intermediate variables, while the second term shows the direct effect of the external input $r_{n}\left(t^{\prime}\right)$ on node output $h_{k}(t)$.

\subsection{Computation of $\frac{\partial^{\dagger 2} E}{\partial r_{n}\left(t^{\prime}\right) \partial \lambda_{m}}$}

Second order derivative of $E$ with respect to the external input $r_{n}\left(t^{\prime}\right)$ and parameter $\lambda_{m}$ can be obtained as follows,

$$
\begin{aligned}
\frac{\partial^{\dagger 2} E}{\partial r_{n}\left(t^{\prime}\right) \partial \lambda_{m}} & =\sum_{r \in J_{o}} \sum_{s \in T_{o}}\left[\frac{\partial^{\dagger}\left(\frac{\partial E}{\partial h_{r}(s)}\right)}{\partial \lambda_{m}} \frac{\partial^{\dagger} h_{r}(s)}{\partial r_{n}\left(t^{\prime}\right)}\right. \\
& \left.+\frac{\partial E}{\partial h_{r}(s)} \frac{\partial^{\dagger 2} h_{r}(s)}{\partial r_{n}\left(t^{\prime}\right) \partial \lambda_{m}}\right] \\
& +\frac{\partial^{\dagger}\left(\frac{\partial E}{\partial r_{n}\left(t^{\prime}\right)}\right)}{\partial \lambda_{m}} \ldots \ldots \ldots \ldots \ldots
\end{aligned}
$$

Putting $P_{1}\left(k, t, r_{n}\left(t^{\prime}\right)\right)=\frac{\partial^{\dagger} h_{k}(t)}{\partial r_{n}\left(t^{\prime}\right)}$, and $P_{2}\left(k, t, r_{n}\left(t^{\prime}\right), \lambda_{m}\right)=$ 
$\frac{\partial^{\dagger} h_{r}(t)}{\partial r_{n}\left(t^{\prime}\right) \partial \lambda_{m}}$, as in the case of the first order derivatives, iterative equation of $P_{2}$ by forward propagation can be obtained by differentiating $P_{1}$ with respect to $\lambda_{m}$,

$$
\begin{aligned}
& P_{2}\left(k, t, r_{n}\left(t^{\prime}\right), \lambda_{m}\right)= \\
& \sum_{j \in J F(k)} \sum_{p \in B(j, k)}\left[\frac{\partial^{\dagger}\left(\frac{\partial h_{k}(t)}{\partial h_{j}\left(t-D_{j k}(p)\right)}\right)}{\partial \lambda_{m}}\right. \\
& \times P_{1}\left(j, t-D_{j k}(p), r_{n}\left(t^{\prime}\right)\right)+\frac{\partial h_{k}(t)}{\partial h_{j}\left(t-D_{j k}(p)\right)} \\
& \left.\times P_{2}\left(j, t-D_{j k}(p), r_{n}\left(t^{\prime}\right), \lambda_{m}\right)\right]+\frac{\partial^{\dagger}\left(\frac{\partial h_{k}(t)}{\partial r_{n}\left(t^{\prime}\right)}\right)}{\partial \lambda_{m}} \\
& P_{2}\left(j, t<t^{\prime}, r_{n}\left(t^{\prime}\right), \lambda_{m}\right)=0 \cdots \cdots \cdots \cdots
\end{aligned}
$$$$
\frac{\partial^{\dagger}\left(\frac{\partial h_{k}(t)}{\partial h_{j}\left(t-D_{j k}(p)\right)}\right)}{\partial \lambda_{m}} \text { and } \frac{\partial^{t}\left(\frac{\partial h_{k}(t)}{\partial r_{n}\left(t^{\prime}\right)}\right)}{\partial \lambda_{m}}
$$$$
\text { in Eq.(18) can be }
$$
calculated by the computation of first order derivatives putting $E^{\prime}=\frac{\partial h_{k}(t)}{\partial h_{j}\left(t-D_{j k}(p)\right)}, E^{\prime \prime}=\frac{\partial h_{k}(t)}{\partial r_{n}\left(t^{\prime}\right)}$ respectively. Substituting $\frac{\partial^{\dagger} h_{r}(s)}{\partial r_{n}\left(t^{\prime}\right)}$ and $\frac{\partial^{\dagger} h_{r}(s)}{\partial r_{n}\left(t^{\prime}\right) \partial \lambda_{m}}$ obtained from Eq.(15) and (18) into Eq.(17), $\frac{\partial^{\dagger 2} E}{\partial r_{n}\left(t^{\prime}\right) \partial \lambda_{m}}$ can be calculated.

\section{Simulation}

In this section, two simulations as examples are carried out to investigate the effectiveness of the proposed method. One is an identification of a cascade connection of nonlinear and linear subsystem. The other is an identification of a real system which describes a hydraulic robot actuator.

\subsection{Example 1}

As an example of investigating the generalization ability, a nonlinear system identification problem was studied, which is a cascade connection of nonlinear subsystem $g_{N}($.$) and linear subsystem g_{L}($.$) shown in Fig.2.$

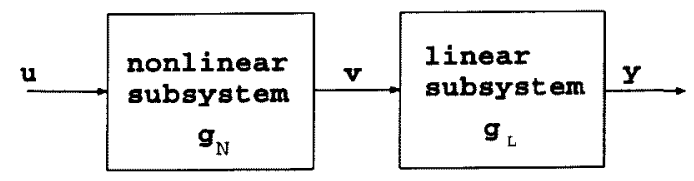

Fig.2 Cascade connection of nonlinear and linear subsystem

The cascade connection system of the nonlinear and linear subsystem was modeled by the ULNs in Fig.1, which has 5 nodes and fully recurrent connections with 1 branch between the nodes and one external input. It was supposed that the output of ULNs can be obtained from node $N_{5}$.

Input value $u(k)$ and output value $y(k)$ of the system to be identified are expressed by Eq.(20).

電学論C, 119 巻 5 号, 平成 11 年

$$
y(k+1)=\left\{\begin{array}{l}
1.34 y(k)-0.277 y(k-2) \\
-0.80 y(k-4)+0.01, \quad u(k) \geq 0 \\
1.34 y(k)-0.277 y(k-2) \\
-0.80 y(k-4)-0.01, \quad u(k)<0
\end{array}\right.
$$

Fig. 3 shows the input and output data for training and testing. Horizontal axis is time, vertical axis is input/output value. In testing data 1 and 2 , the order of sine function, rectangular function and random number for input $u(k)$ is changed from the order of the training data. Fig. 4 shows the simulation results when $k_{d}=0.0$ 0.005 and 0.01 respectively.

As simulation conditions, the following are used, nonlinear function $f(x)=A \frac{1-e^{-x}}{1+e^{-x}}$ while $A=1.5$, initial value of parameter $\lambda_{m}$ random numbers in $(-1.0,+1.0)$, learning coefficient of $\lambda_{m} \gamma=0.00002$, time delays random numbers in $(1,10)$. And identification error $E$ is the root square error. The regularization parameter $k_{d}$ is set at $0.0,0.005$ and 0.01 respectively. The number of training is 100000 .
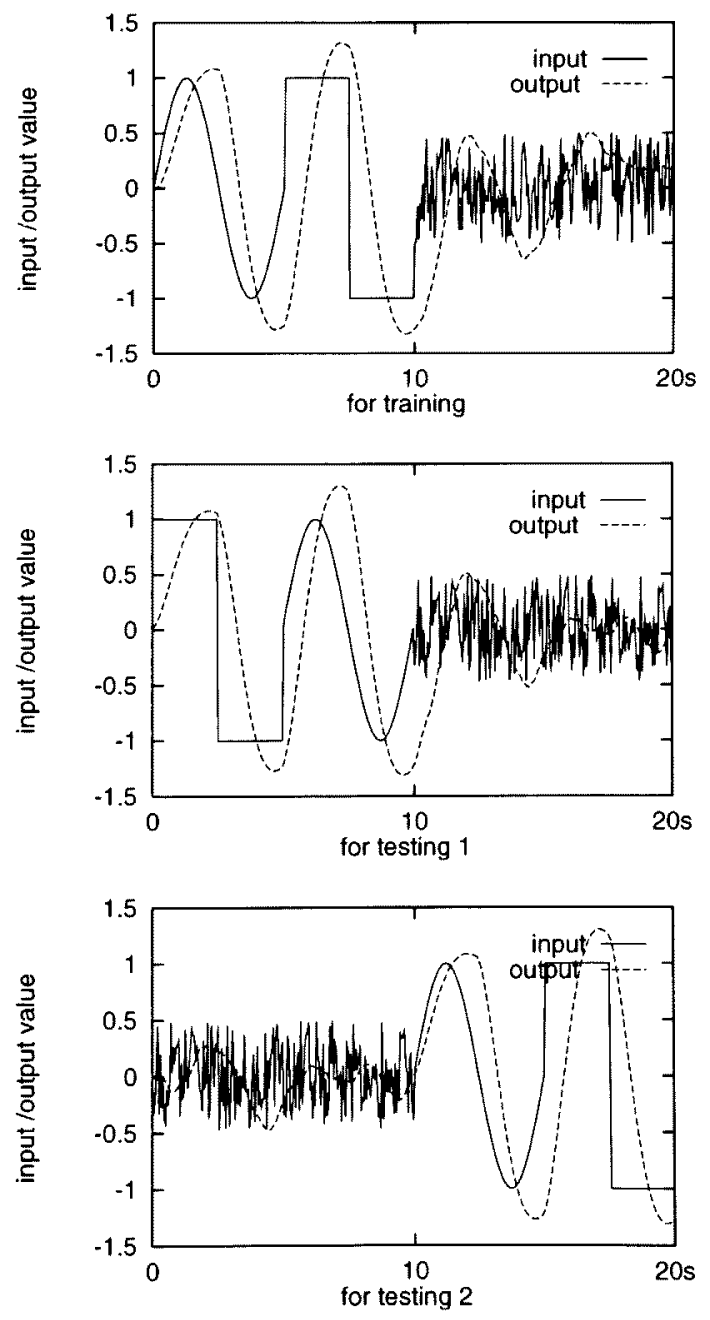

Fig.3 Input and output data of a cascade connection system 
From simulation results, the following conclusions can be obtained:

(1) By selecting an appropriate regularization parameter $k_{d}$, we can improve the generalization ability. Specifically, when $k_{d}$ is assigned a relatively large value, the deviation of $E$ caused by the change of the external input can be curbed quite sufficiently so that the generalization ability is improved.

(2) The generalization ability of not only the static systems but also the dynamic systems can be improved by the proposed method in which the second order derivatives are effectively used to train the parameters.
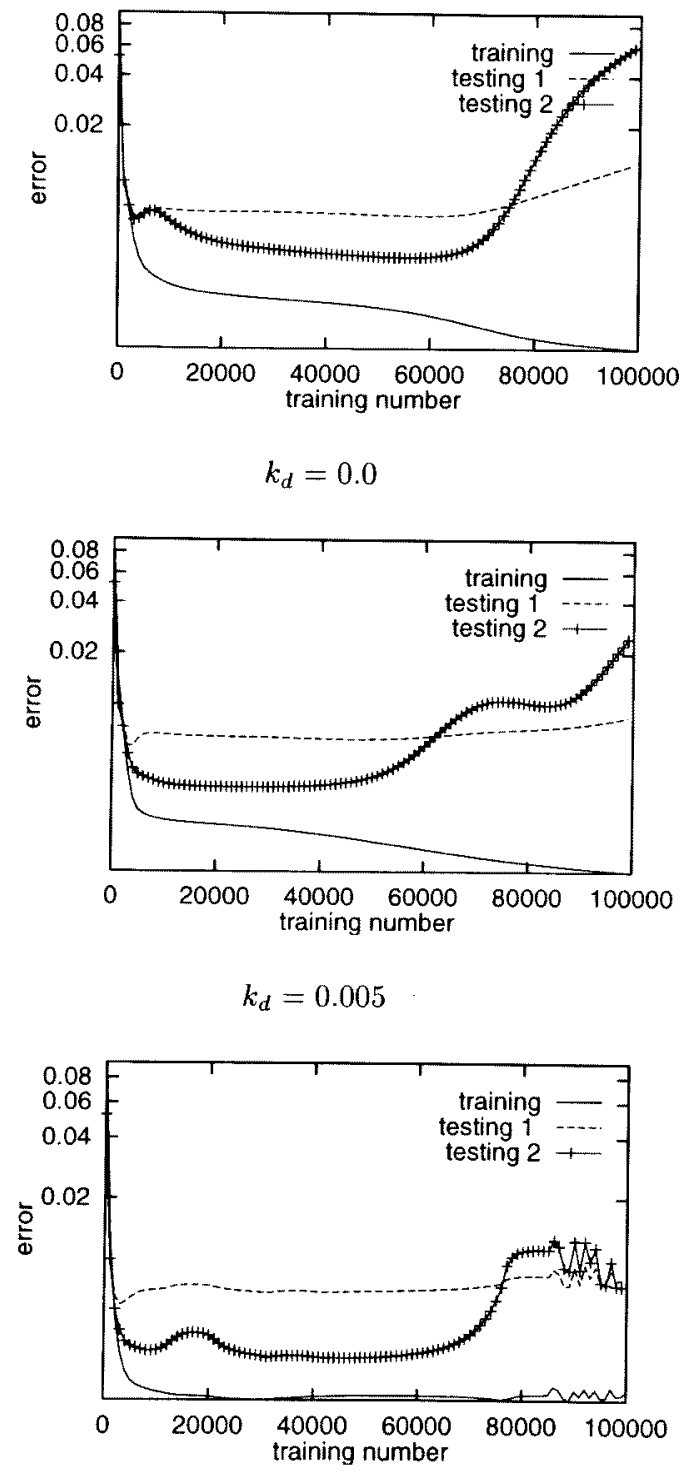

$$
k_{d}=0.01
$$

Fig 4. Results of root square error for various regularization parameters in a cascade connection system

\subsection{Example 2}

So as to investigate the effectiveness of the proposed method, a real hydraulic robot actuator system is used in this section. The goal is to model the dynamics of a hydraulically controlled robot arm. The position of the robot arm is controlled by the oil pressure in the cylinder which, in turn, is controlled by the size of the valve through which the oil flows. The position of the value and the oil pressure at time $t$ are noted by $u(t)$ and $y(t)$ respectively. A sample of 1024 pairs of $\{y(t), u(t)\}$ was registered ${ }^{(19)}$. We divide it into two equal parts for estimating and for validating our model. The estimation data is depicted in Fig.5.

As simulation conditions, the following are assumed, the number of nodes is 10 , the number of branches between nodes is 1 , nonlinear function is $f(x)=A \frac{1-e^{-x}}{1+e^{-x}}$ while $A=1.5$, initial value of parameter $\lambda_{m}$ is random numbers in $(-1.0,+1.0)$, learning coefficient of $\lambda_{m}$ is $\gamma=0.00002$, time delays are random numbers in $(1,3)$, identification error $E$ is the root square error, regularization parameter $k_{d}$ is set at $0.0,0.00005$ and 0.005 respectively, the number of training is 10000 .
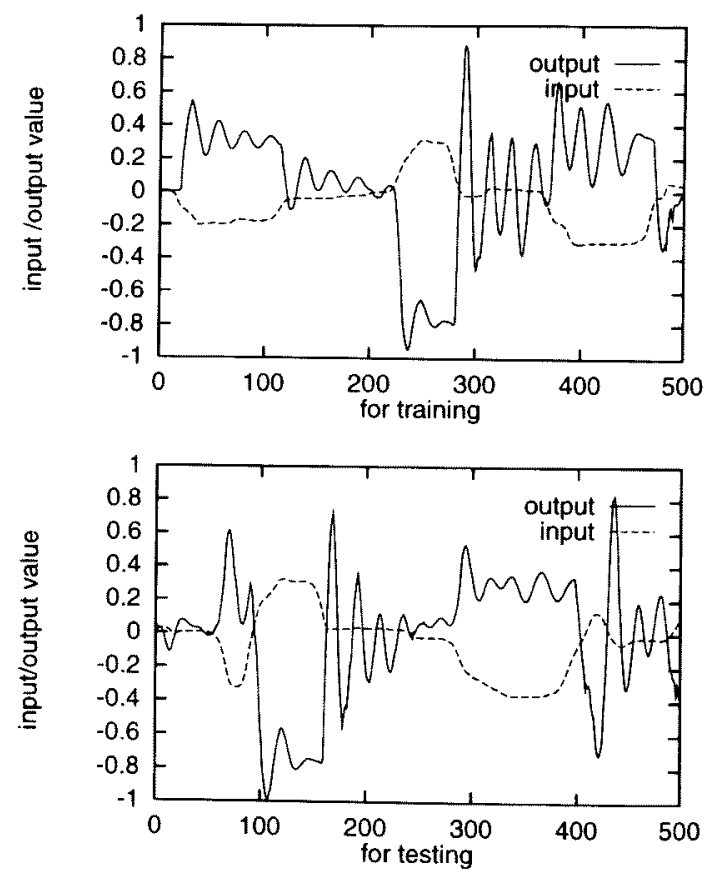

Fig.5 Input and output data of a robot system

Fig.6 shows the simulation results when $k_{d}=0.0$, 0.00005 and 0.005 respectively. The conclusion is that by selecting an appropriate $k_{d}$ the error of using the second order derivatives is less than that obtained without using the second order derivatives. So, it can be seen that regularization parameter $k_{d}$ is a very important factor for training.

\section{Conclusions}

In this paper, a method based on regularization tech- 
nique is proposed for improving the generalization ability of the dynamic networks. The main points of the proposed method are as follows:

(1)direct implementation of regularization technique

(2)use of second order derivatives of Universal Learn ing Networks
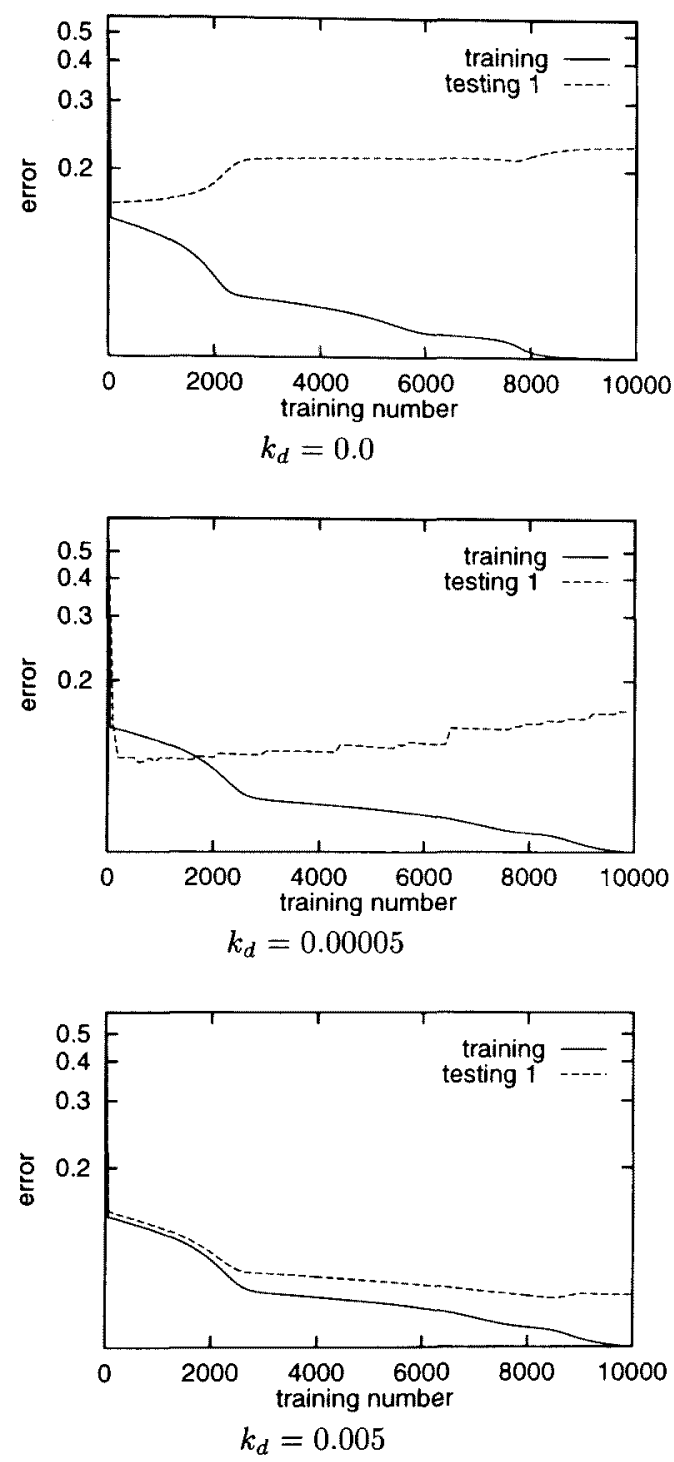

Fig.6 Results of root square error for various regularization parameters in a robot system

By using these techniques, redundant parameters of networks are taken into account for improving the generalization ability of the networks.

Through simulations of the identification of nonlinear dynamic systems, it has been turned out that the proposed method can improve the generalization ability of neural networks sufficiently. It has also been clarified that regularization parameter $k_{d}$ is a very important factor to secure the satisfactory results, so one of the problems to be solved in a future is to find out how to select an appropriate $k_{d}$ depending on the systems.

(Manuscript received June 16, 1998, revised January $6,1999)$

\section{References}

(1) K. Hirasawa, M. Ohbayshi, and J. Murata "Universal learn ing network and computation of its higher order derivative," in Proc of IEEE International Conference on Neural Net works, vol. 3 , pp. 1273-1277, 1995

(2) K. Hirasawa, M. Ohbayahsi, M. Koga, and M. Harada "Forward propagation universal learning network," in Proc of IEEE International Conference on Neural Networks, pp. $353-358,1996$

(3) K. Hirasawa, J. Hu, M. Ohbayashi, and J. Murata, "Comput ing higher order derivatives in universal learning networks," Journal of Advanced Computational Intelligence, vol. 2, no. 2 , pp. $47-53,1998$

(4) G. Hinton, Connectionist Learning Procedure, Machine Learning. The MIT Press, 1990.

(5) M. Ishikawa and K. Yoshino, "Automatic task decomposition in modular networks by structure learning with forgetting," in Proc. of IEEE International Joint Conference on Neural Networks, pp. 1345 -1348, 1993.

(6) A. Weigend, D. Rumelhart, and B. Huberman, "Generalization by weight-elimination with application to forgetting," Advances in Neural Information Processing Systems, vol. 3 , pp. 875-882, 1991

(7) T. Poggio and F. Givosi, "Regularization algorithms for learning that are equivalent to multilayer networks," Science, vol. 247 , pp. $978-987,1990$.

(8) M. Han, K. Hirasawa, M. Ohbayashi, and H. Fujita, "Modeling dynamic systems using Universal Learning Network," in Proc. of IEEE International Conference on Systems, Man and Cybernetics, pp. 1172-1177, 1996.

(9) M. Han, K. Hirasawa, M. Ohbayashi, and H. Fujita, "Generalization ability of modeling dynamic systems using Universal Learning Network," in Proc. of the 11th IFAC Symposium on System Identification, pp. 1143-1148, 1997.

(10) T. Poggio and F. Givosi, "Networks for approximation and learning," Proceedings of the IEEE, vol. 78 , no. 9, pp. 14811497,1990

(11) A. Tikhonov, "Solution of incorrectly formulated problems and the regularization method," Soviet Meth. Dokl, vol. 4 , pp. $1035-1038,1963$

(12) A. Tikhonov and V. Arsenin, Solutions of Ill-posed Problems. W.H.Winston, Washington, D.C, 1977.

(13) V. Morozov, Methods for Solving Incorrectly Posed Problems. Springer-Verlag, Berlin, 1984

(14) M. Bertero, "Regularization methods for linear inverse problems," in Inverse Problems (C. Talenti, ed.), Springer-Verlag, Berlin, 1986.

(15) F. Girosi and T. Poggio, "Networks and the best approximation property," Biological Cybernetics, vol. 63, pp. 169-176 1990.

(16) J. Sjöberg and L. Ljung, "Overtraining, regularization and searching for a minimum, with application to neural networks," INT. J. Control, vol. 62, no. 6, pp. 1391-1407, 1995

(17) M. Ishikawa, "Structure learning with forgetting," Neural Networks, vol. 9, no. 3, pp. 509-521, 1996.

(18) P. Werbos, Beyond Regression: New Tools for Prediction and Analysis in the Behavior Science. $\mathrm{PhD}$ thesis, Harvard University, 1974.

(19) J. Sjöberg, Q. Zhang, L. Ljung, A. Benveniste, B. Deglon, P. Glorennec, H. Hjalmarsson, and A. Juditsky, "Nonlinear black-box modeling in system identification: a unified overview," A utomatica, vol. 31, no. 12, pp. 1691-1724, 1995. 


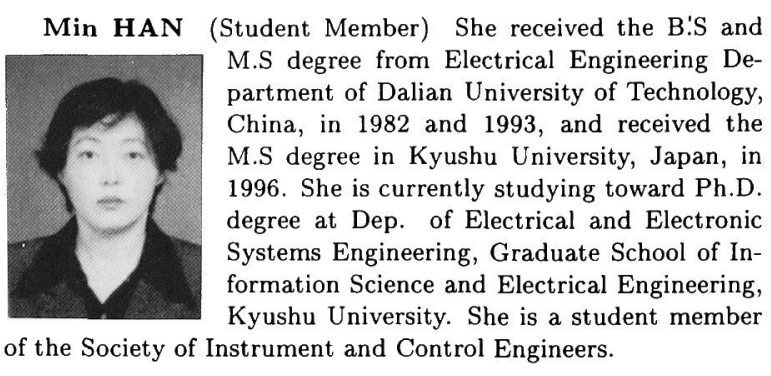

Kotaro HiRASAWA (Member) He received the M.S. degree in Electrical Engineering from Kyushu University in 1966. From April 1966, he served in Hitachi Lab. of Hitachi Ltd., and in 1989 he was a vice president of Hitachi Lab.. From August 1991 to November 1992, he served in Omika Factory of Hitachi Ltd.. Since December 1992, he has been a professor in the faculty of Engineering, Kyushu University. Now he belongs to the Graduate School of Information Science and Electrical Engineering, Kyushu University. Dr. Hirasawa is a member of the Society of Instrument and Control Engineers, a member of IEEE.

Jinglu HU (Member) He received the M.Sci. degree in

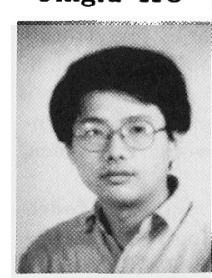
1986 from Zhongshan University, China and the Ph.D degree in 1997 from Kyushu Institute of Technology. From 1986 to 1993, he was a Research Associate and then a Lecturer in Zhongshan University. Since 1997, he has been Research Associate at Kyushu University. His current research interests are learning network theory, system identification and their applications. Dr. Hu is a member of the Society of Instrument and Control Engineers.

Junichi MURATA (Member) Dr Junichi MURATA grad-

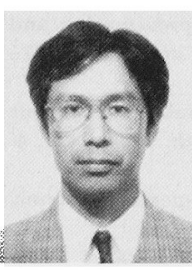

uated from Kyushu University in 1981. He received Master of Engineering and Doctor of Engineering degrees from Kyushu University in 1983 and 1986, respectively. He then became a Research Associate and an Associate Professor at Faculty of Engineering, Kyushu University. Now he is an Associate Professor at Graduate School of Infomation Science and Electrical Engieering, Kyushu University. His current research interests are neural networks, self-organizing systems and their applications to control and identification. He is a member of SICE, ISCIE and IEEE. 